
G. Perinetti
L. Perillo
L. Franchi
R. Di Lenarda
L. Contardo

\section{Maturation of the middle phalanx of the third finger and cervical vertebrae: a comparative and diagnostic agreement study}

\author{
Authors' affiliations: \\ G. Perinetti, R. Di Lenarda, L. Contardo, \\ Department of Medical, Surgical and \\ Health Sciences, School of Dentistry, \\ University of Trieste, Trieste, Italy \\ L. Perillo, Department of Orthodontics, \\ School of Dentistry, Second University of \\ Naples, Naples, Italy \\ L. Franchi, Department of Orthodontics, \\ School of Dentistry, University of \\ Florence, Florence, Italy \\ L. Franchi, Department of Orthodontics \\ and Pediatric Dentistry, School of \\ Dentistry, The University of Michigan, \\ Ann Arbor, MI, USA

\section{Correspondence to:} \\ G. Perinetti \\ Struttura Complessa di Clinica Odontoia- \\ trica e Stomatologica \\ Ospedale Maggiore \\ Piazza Ospitale 1 \\ 34129 Trieste \\ Italy \\ E-mail: G.Perinetti@fmc.units.it
}

\section{Date:}

Accepted 14 August 2014

DOI: 10.1111/ocr.12052

(C) 2014 John Wiley \& Sons A/S.

Published by John Wiley \& Sons Ltd
Perinetti G., Perillo L., Franchi L., Di Lenarda R., Contardo L. Maturation of the middle phalanx of the third finger and cervical vertebrae: a comparative and diagnostic agreement study

Orthod Craniofac Res 2014; 17: 270-279. (C) 2014 John Wiley \& Sons A/S. Published by John Wiley \& Sons Ltd

\section{Structured Abstract}

Objective - Diagnostic agreement on individual basis between the third middle phalanx maturation (MPM) method and the cervical vertebral maturation (CVM) method has conjecturally been based mainly on overall correlation analyses. Herein, the true agreement between methods according to stage and sex has been evaluated through a comprehensive diagnostic performance analysis.

Subjects and methods - Four hundred and fifty-one Caucasian subjects were included in the study, 231 females and 220 males (mean age, $12.2 \pm 2.5$ years; range, $7.0-17.9$ years). The $X$-rays of the middle phalanx of the third finger and the lateral cephalograms were examined for staging by blinded operators, blinded for MPM stages and subjects' age. The MPM and CVM methods based on six stages, two pre-pubertal (1 and 2), two pubertal (3 and 4), and two post-pubertal (5 and 6), were considered. Specifically, for each MPM stage, the diagnostic performance in the identification of the corresponding CVM stage was described by Bayesian statistics.

Results - For both sexes, overall agreement was $77.6 \%$. Most of the disagreement was due to 1 stage apart. Slight disagreement was seen for the stages 5 and 6, where the third middle phalanx shows an earlier maturation.

Conclusions - The two maturational methods show an overall satisfactorily diagnostic agreement. However, at post-pubertal stages, the middle phalanx of the third finger appears to mature earlier than the cervical vertebrae. Post-pubertal growth phase should thus be based on the presence of stage 6 in MPM.

Key words: cervical vertebrae; diagnosis; finger phalanges; growth 


\section{Introduction}

When dealing with skeletal disharmonies for treatment efficiency reasons, the precise identification of skeletal maturity, that is, the growth phase, with particular regard to the onset of the pubertal growth spurt, is required $(1,2)$. Several indices have been proposed to identify the skeletal maturation phases (1-6). The most commonly used are the radiography-based, handwrist maturation [for review, see (7)] and cervical vertebral maturation (CVM) [for review, see (1)] methods.

Several studies have previously evaluated the relationship between these two methods [for review, see (8)] generally reporting a high degree of correlation. However, all of these previous studies were hampered by the use of a specific CVM recording (9) not consistent with the described methods $(1,10)$ validated in clinical trials (11) or by lack of an analysis of diagnostic agreement in individual subjects $(8,12)$. Indeed, a high correlation coefficient does not necessarily prove a diagnostic agreement in individual subjects, as was recently showed for dental maturation (13). This issue may be addressed by a dedicated diagnostic performance analysis that is, however, still missing. Moreover, very few studies (14-16) have specifically been focused on the correlations between the middle phalanx maturation (MPM) of the third finger and the CVM method. The results of these investigations were further limited by the lack of an accurate recording of the repeatability of the measurements of both maturational methods (14), or because only male subjects were included (15).

This study was designed to address the following issues: 1) Does the middle phalanx of the third finger and cervical vertebral maturations have satisfactorily diagnostic agreement? and 2) If disagreement is seen, how is this structured among the different stages or sexes? This study ultimately verified whether the MPM method, as proposed herein, may be proposed as a valid indicator of growth phase in individual subjects.

\section{Materials and methods}

Study population and design

The databases between January 2008 and August 2013 of the Sections of Stomatology of the Department of Medical, Surgical and Health Sciences, University of Trieste, and of the Department of Oral Sciences, Second University of Naples, were screened. This study included subjects who were seeking orthodontic treatment. Signed informed consent was obtained from the parents of the subjects prior to study entrance, and the protocol was reviewed and approved by the local ethical committee. In particular, an $\mathrm{X}$-ray of the middle phalanx of the middle finger and a lateral cephalogram is taken as part of the routine clinical recording. The following inclusion criteria were applied: 1) age between 7 and 18 years; 2) absence of anomalies of either the fingers or the vertebrae; 3) good general health with the absence of any nutritional problems; 4) no history of trauma at the cervical region or right hand; and 5) Caucasian ethnicity. A total of 451 subjects (231 females and 220 males) were included in the study (mean age, $12.2 \pm 2.5$ years; range, $7.0-17.9$ years). In a posteriori power analysis, sample size of 278 subjects is enough to detect an agreement between the maturational methods as low as $30 \%$ considering a relative error (the difference between the estimated and true reliability), as low as $20 \%$ with a power of 0.8 , and an alpha set at 0.05 (17).

\section{Radiographic recordings}

The radiographic recording of the middle phalanx of the middle finger was performed as previously reported (18). Briefly, the patients were instructed to place their right hand with the palm downward on a flat table and with the third finger straight and centered on a standard $3 \times 4 \mathrm{~cm}$ periapical sensor (Dürr Dental, Bietigheim-Bissingen, Germany). The cone of the dental X-ray machine (Kodak 2200 intraoral x-ray system; Eastman Kodak Company, Rochester, NY, USA) was positioned in light contact with 
the middle phalanx and perpendicular to the dental X-ray sensor. Settings were of $70 \mathrm{kV}$ and $7 \mathrm{~mA}$ with an exposure time of $0.097 \mathrm{~s}$. An automatic developer (VistaScan PERIO; Dürr Dental) was used for film processing. A dedicated X-ray machine (KODAK 8000C; Eastman Kodak Company) was employed for the recording of lateral head cephalogram. Settings were of $73-77 \mathrm{kV}$, $12 \mathrm{~mA}$, with an exposure time of $0.80 \mathrm{~s}$. Radiographs of low quality were excluded.

\section{Middle phalanx maturation (MPM) method}

The MPM method as proposed herein comprises 6 stages [middle phalanx stages, (MPS)], as shown in Fig. 1. Definitions of the stages were based on previous descriptions by Fishman (3), Hägg and Taranger (5), and Rajagopal and Kansal (14), with modifications:

\section{MPS1}

When the epiphysis is narrower than the metaphysis, or when the epiphysis is as wide as metaphysis (5), but with both tapered and rounded lateral borders (14) (Fig. 1, MPS1b). Epiphysis and metaphysis are not fused. This stage was earlier reported as MP3-F and described to be attained more than 1 year before the onset of the pubertal growth spurt (5).
MPS2

When the epiphysis is at least as wide as the metaphysis (5) with sides increasing thickness and showing a clear line of demarcation at right angle (5). In case of asymmetry between the two sides, that is, one typical of MPS2 and the other less mature, the former is used to assign the stage. This stage was earlier reported as SMI2 (3) or as MP3-FG described to be attained 1 year before the onset of the pubertal growth spurt (5).

MPS3

When the epiphysis is either as wide as or wider than the metaphysis (5) with lateral sides showing an initial capping toward the metaphysis (5). In case of asymmetry between the two sides, for example, one typical of MPS3 and the other less mature, the former is used to assign the stage. Epiphysis and metaphysis are not fused. This stage was earlier reported as SMI6 (3) or as MP3-G, both described to be attained at coincidence of the pubertal growth spurt (5).

MPS4

When the epiphysis begins to fuse with the metaphysis (5) although contour of the former is still clearly recognizable. Both sides of the epiphysis form obtuse angle to distal border,

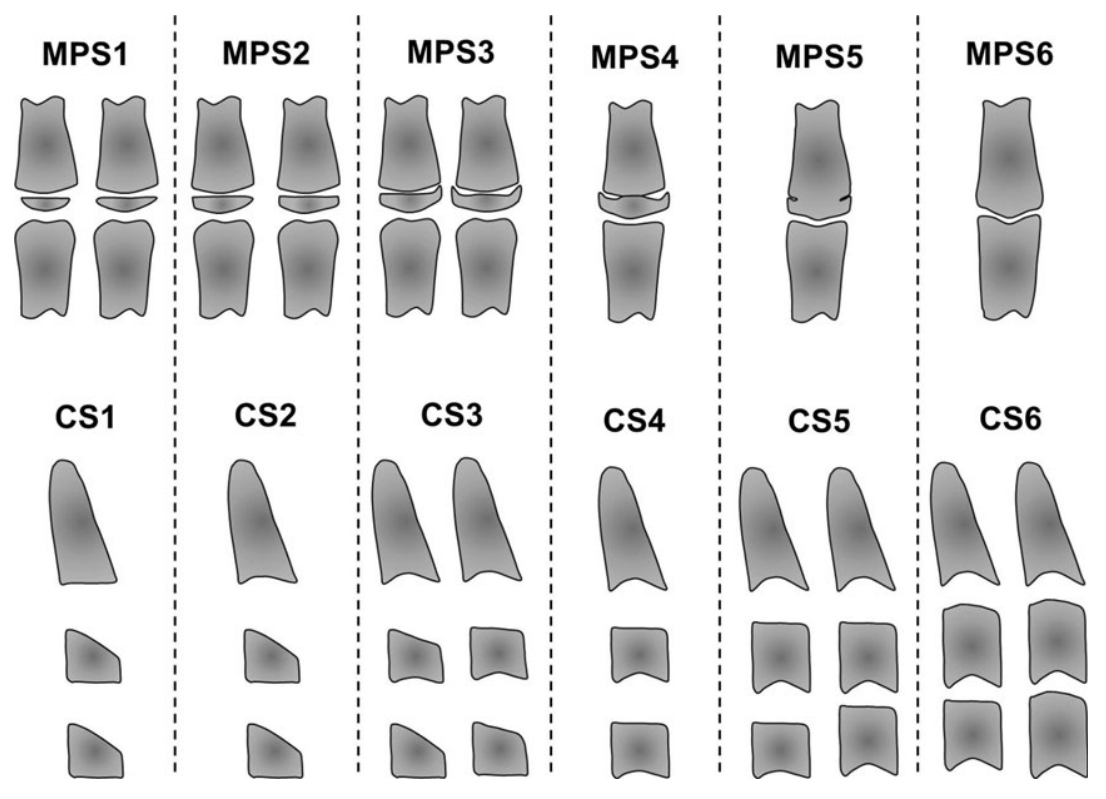

Fig. 1. The third middle phalanx (upper) and cervical vertebral (lower) maturational stages. MPS, third middle phalanx maturational stage; CS, cervical vertebral maturational stage. 
and the capping is still clearly detectable. This stage was earlier reported as MP3-H and described to be attained after the pubertal growth spurt, that is, during the deceleration of the curve of growth (5).

MPS5

When the epiphysis is mostly, but not completely fused with the metaphysis (5), and the distal contour of the former begins to be less clearly recognizable. This specific stage was initially proposed by Rajagopal and Kansal (14) and reported as MP3-HI and was reported to be attained toward the end of the pubertal growth spurt (14).

MPS6

When the epiphysis totally fused with the metaphysis (5), and the distal contour of the former is not recognizable. This stage was earlier reported as SMI10 (3) or as MP3-I, both described to be attained at the end of the pubertal growth spurt (5).

An experienced orthodontist (GP), who was blinded to the CVM stages, assessed the MPM stages.

\section{Cervical vertebral maturation method}

The CVM method as initially proposed by Hassel and Farman (10), and subsequently modified according to Baccetti et al. (1), comprises 6 stages (CS) as shown in Fig. 1 and as briefly defined as follows:

CS1

When the lower borders of the second, third, and fourth vertebrae (C2, C3, and C4) are flat, and the bodies of $\mathrm{C} 3$ and $\mathrm{C} 4$ are trapezoid in shape. This stage has been reported to be attained at least 2 years before the pubertal growth spurt.

CS2

When only the lower border of C2 is concave, and the bodies of C3 and C4 are trapezoid. This stage has been reported to be attained 1 year before the growth spurt.
CS3

When the lower borders of both C2 and C3 have concavities, and the bodies of C3 and C4 are either trapezoid or rectangular horizontal in shape. This stage has been reported to occur in coincidence with the onset of the pubertal growth spurt, that is, acceleration curve of growth.

CS4

When the lower borders of $\mathrm{C} 2-\mathrm{C} 4$ have concavities, and the bodies of both $\mathrm{C} 3$ and $\mathrm{C} 4$ are rectangular horizontal. This stage has been described to be attained at coincidence of the pubertal growth spurt, but after the peak height velocity, that is, during the deceleration curve of growth.

CS5

When the lower borders of $\mathrm{C} 2-\mathrm{C} 4$ have concavities, and at least one or both of the bodies of C3 and C4 is square. This stage has been reported to occur 1 year after the growth spurt.

CS6

When the lower borders of $\mathrm{C} 2-\mathrm{C} 4$ have concavities, and at least one or both of C3 and C4 are rectangular vertical. This stage has been reported to occur at least 2 years after the growth spurt.

The lateral cephalograms were cropped to include $\mathrm{C} 2-\mathrm{C} 4$ and to eliminate any additional information, such as stage of dentition that might have biased the staging. An experienced orthodontist (LC) with 5-year experience in the CVM method including training with the developers of this staging, blinded to the MPM stages and subjects' age, assessed the CVM stages.

\section{Statistical analysis}

All these analyses were performed for each sex separately as well as for the whole sample. Mean ages of the subjects, clustered according to each stage of either maturational methods, have been plotted, and within each MPM stage, the prevalence of the CVM stages was calculated. To determine the degree of correlation between the two maturational indices, the Spearman rank correlation coefficient was used. The diagnostic agree- 
ment between the MPM and CVM methods was assessed by a linearly weighted $\kappa$ coefficient (19).

Moreover, a dedicated diagnostic performance analysis, that is, Bayesian statistics, was also performed to establish the diagnostic performance of each MPM stage for the identification of each corresponding CVM stage. This analysis included sensitivity, specificity, positive and negative predictive values, accuracy, and positive likelihood ratio (LHR) (20). A threshold of a positive LHR of $\geq 10$ (21) was considered for assessment of satisfactory reliability of any MPM stage for the identification of any of the CVM stages, that is, satisfactory diagnostic agreement.

The percentage agreement and weighted $\kappa$ statistics were calculated for evaluation of the intra-examiner agreement. For appraisal of the stages of MPM and CVM, the intrarater weighted $\kappa$ coefficients calculated on 30 pairs of recordings randomly selected were $>0.92$.

SPSS software 13.0 (SPSS $^{\circledR}$ Inc., Chicago, IL, USA), MedCalc ${ }^{\circledR}$ software 12.3.3.0 (MedCalc Software, Mariakerke, Belgium) and the interactive Stats Calculator (http://ktclearinghouse.ca/cebm/ toolbox/statscalc) were used to perform the statistical analyses. A $p$ value $<0.05$ was considered as significant.

\section{Results}

The comparative mean ages of the subjects for each MPM or CVM stage according to the sexes are shown in Fig. 2. Mean ages were very similar with few exceptions for males at stage 5 , in which the differences were about 0.6 years. For both the maturational methods, the differences in chronological ages between two consecutive stages from 2 to 5 ranged from about 0.6 to 1.5 years for both sexes. Irrespective of the maturational method, females attained stages $2-6$ generally 1 year earlier than males. Clinical examples for each MPM and CVM stages are shown in Fig. 3.

Of the whole sample, 350 subjects $(77.6 \%)$ showed a full agreement between the two maturational stages, 89 subjects $(19.7 \%)$ showed a one-stage-apart disagreement, while in only 12 cases $(2.7 \%)$, a two-stage-apart disagreement
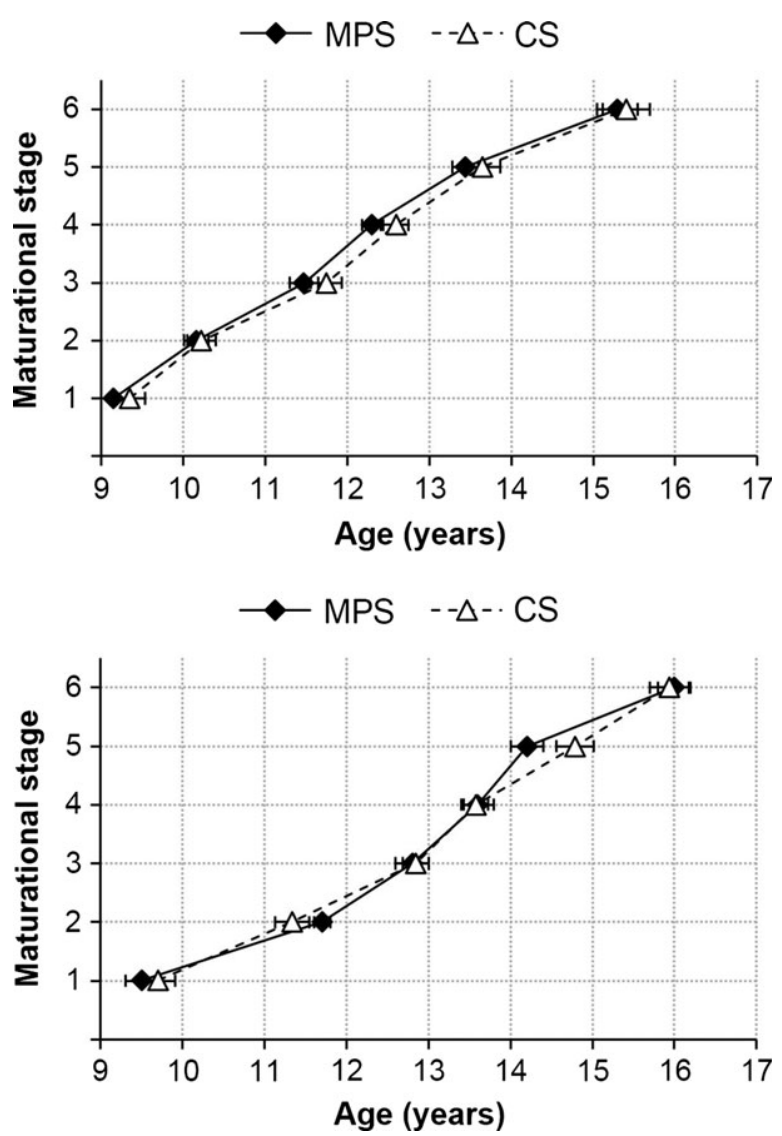

Fig. 2. Chronological ages among the different third finger middle phalanx and cervical vertebral maturational stages for females (upper) and males (lower). Data are presented as mean \pm standard error of the mean. MPS, third middle phalanx maturational stage; CS, cervical vertebral maturational stage. Females, $\mathrm{n}=231$; males, $\mathrm{n}=220$.

was seen. The total agreements were 77.9 and $77.3 \%$ for females and males, respectively. The correlation coefficient between the two maturational methods was $0.953(p<0.001)$ for the whole sample, and of $0.953(p<0.001)$ and 0.952 $(p<0.001)$ for females and males, respectively. The weighted $\kappa$ coefficient (95\% CI) for the diagnostic agreement among the different MPM and CVM stages was $0.88(0.85-0.90)$ for the whole sample, and of $0.88(0.84-0.91)$ and $0.87(0.84-$ 0.91) for females and males, respectively.

Detailed relative distributions of the different MPM stages according to CVM stages for females and males are summarized in Table 1. The percentage of exact agreement of the MPM stages with the corresponding CVM stages ranged between $68.3 \%$ (MPS2/CS2) and $97.6 \%$ (MPS1/CS1) for females, and between $57.7 \%$ (MPS5/CS5) and 90.6\% (MPS1/CS1) for males. 
Fig. 3. Clinical examples from six subjects of this study for the third middle phalanx and cervical vertebral maturational stages. MPS, third middle phalanx maturational stage; $C S$, cervical vertebral maturational stage. Note that pubertal middle phalanx maturation stages 3 and 4 may or may not show undulation of the border of the metaphysis.

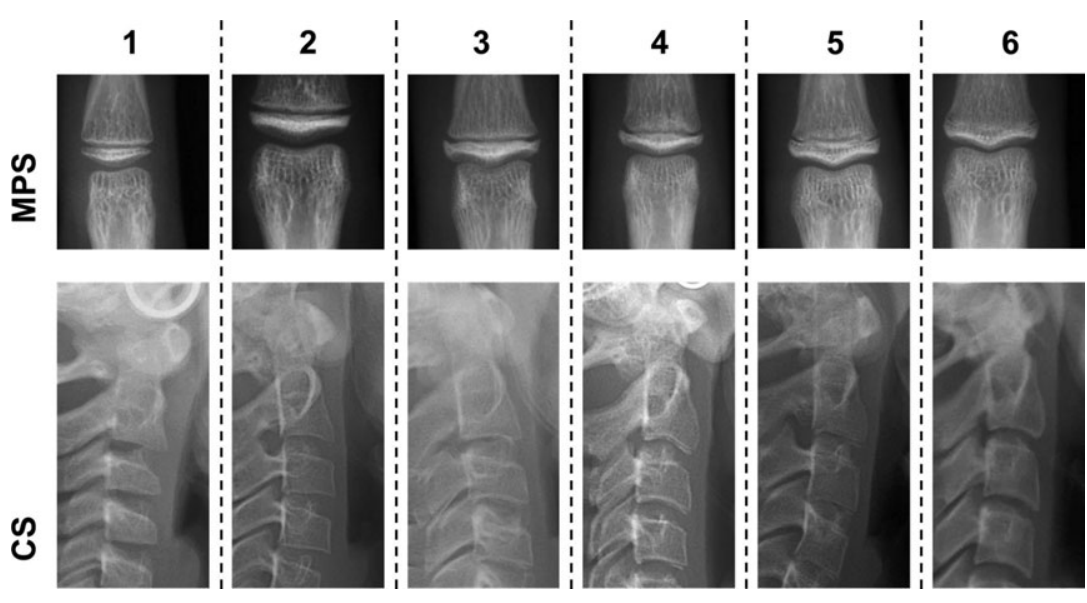

Table 1. Relative distributions of the different third middle phalanx maturational stages according to cervical vertebral maturational stages for females and males

\begin{tabular}{|c|c|c|c|c|c|c|c|c|}
\hline \multirow[b]{2}{*}{ Sex } & \multirow{2}{*}{$\begin{array}{l}\text { Third finger middle } \\
\text { phalanx maturational stage }\end{array}$} & \multicolumn{6}{|c|}{ Cervical vertebral maturational stage } & \multirow[b]{2}{*}{ Total } \\
\hline & & CS1 & CS2 & CS3 & CS4 & CS5 & CS6 & \\
\hline \multirow[t]{6}{*}{ Females } & MPS1 & $97.6 \%(41)$ & $2.4 \%(1)$ & - & - & - & - & 42 \\
\hline & MPS2 & $24.4 \%(10)$ & $68.3 \%(28)$ & $4.9 \%(2)$ & $2.4 \%(1)$ & - & - & 41 \\
\hline & MPS3 & $3.1 \%(1)$ & $15.6 \%(5)$ & $75.0 \%(24)$ & $6.3 \%(2)$ & - & - & 32 \\
\hline & MPS4 & - & - & $13.5 \%(5)$ & $78.4 \%(29)$ & $8.1 \%(3)$ & - & 37 \\
\hline & MPS5 & - & - & $5.1 \%(2)$ & $15.4 \%(6)$ & $76.9 \%(30)$ & $2.6 \%(1)$ & 39 \\
\hline & MPS6 & - & - & - & $5.0 \%(2)$ & $25.0 \%(10)$ & $70.0 \%(28)$ & 40 \\
\hline \multirow[t]{6}{*}{ Males } & MPS1 & $90.6 \%(58)$ & $21.1 \%(6)$ & - & - & - & - & 64 \\
\hline & MPS2 & $21.1 \%(8)$ & $73.7 \%(28)$ & $5.3 \%(2)$ & - & - & - & 38 \\
\hline & MPS3 & - & $6.5 \%(2)$ & $77.4 \%(24)$ & $16.1 \%(5)$ & - & - & 31 \\
\hline & MPS4 & - & - & $7.1 \%(2)$ & $75.0 \%(21)$ & $10.7 \%(3)$ & $7.1 \%(2)$ & 28 \\
\hline & MPS5 & - & - & $7.7 \%(2)$ & $26.9 \%(7)$ & $57.7 \%(15)$ & $7.7 \%(2)$ & 26 \\
\hline & MPS6 & - & - & - & $6.1 \%(2)$ & $21.2 \%(7)$ & $72.7 \%(24)$ & 33 \\
\hline
\end{tabular}

MPS, third middle phalanx maturational stage; CS, cervical vertebral maturational stage.

Data are presented as percentage $(n)$ cases of each MPS within each CS. - , no cases. Females, $n=231 ;$ males, $n=220$.

The other diagnostic performance parameters of different MPM stages and the corresponding CVM stages are summarized in Table 2. Sensitivity ranged between $72.5 \%$ (MPS4/CS4) and 96.6\% (MPS6/CS6) for females, and between $60.0 \%$ (MPS4/CS4 and MPS5/CS5) and 87.9\% (MPS1/CS1) for males. Specificity values were all above $90 \%$ for females and males.

Positive predictive values ranged between 68.3\% (MPS2/CS2) and 97.6\% (MPS1/CS1) for females, and between $57.7 \%$ (MPS5/CS5) and 90.6\% (MPS1/CS1) for males. Negative predictive values and the accuracy values were all above
$90 \%$ for both the sexes. Finally, positive LHRs ranged between 12.5 (MPS2/CS2) and 131.3 (MPS1/CS1) for females, and between 10.7 (MPS5/CS5) and 22.5\% (MPS1/CS1) for males.

\section{Discussion}

The present study reported on the diagnostic agreement between the different stages of maturation of the middle phalanx of the third finger and the cervical vertebral on a population of Caucasic growing subjects. 


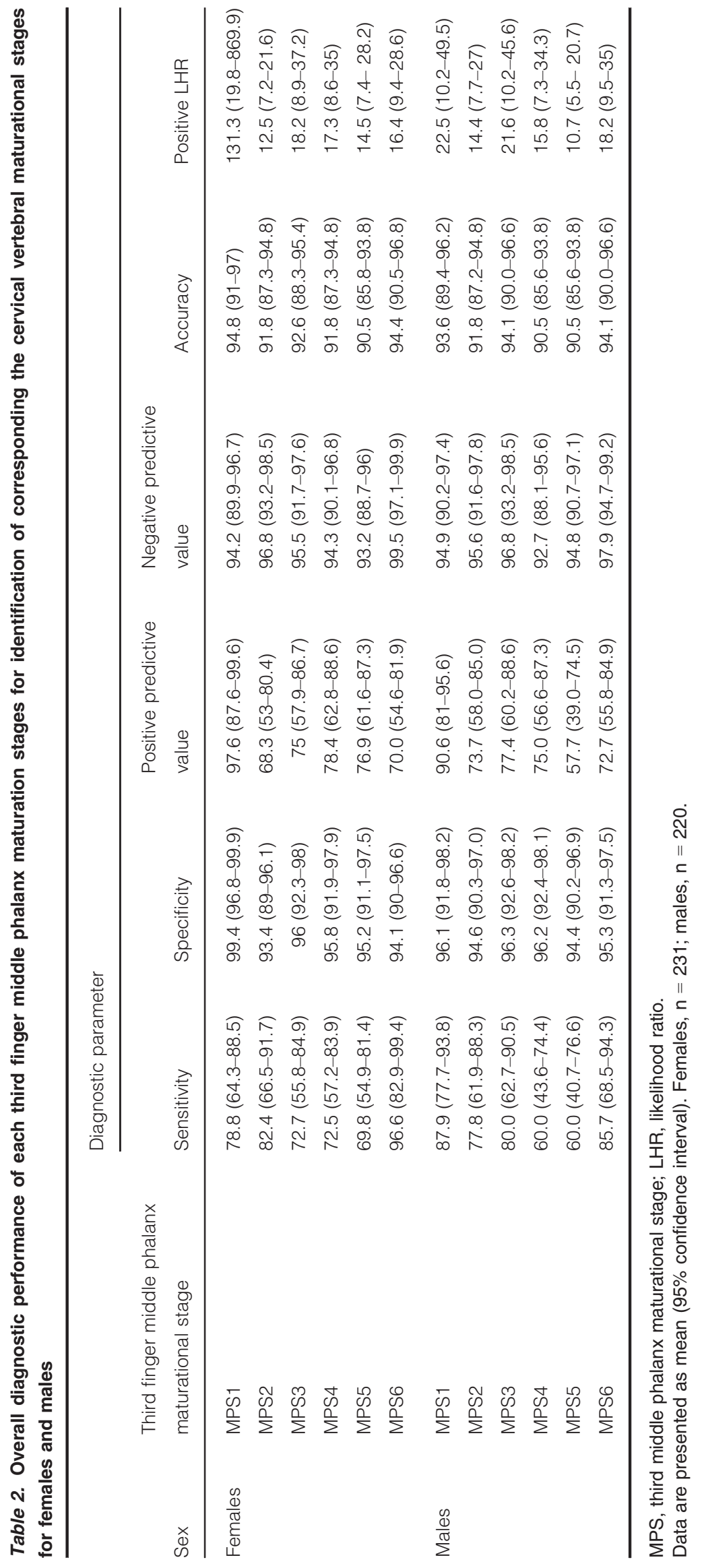


The CVM method has been correlated with both the statural and the mandibular growth spurt (22, 23), and even with levels of biomarkers of growth $(24,25)$. A randomized clinical trial on functional treatments has proved the validity of this method in terms of skeletal outcome (11). Of note, previous studies reported a low to good reproducibility of the CVM method with weighted $\kappa$ values ranging from 0.36 to 0.79 according to the different raters (26). A later investigation, using the same sample and raters, reported that the assignment of the shape of the bodies of C3 and C4 is the least reproducible part of the CVM staging (27). In spite of this evidence, the intrarater reproducibility obtained in the present study was satisfactory with weighted $\kappa$ of 0.92 . The high reproducibility seen herein was likely due to the extensive training of the rater.

In addition to specific training, the CVM method also requires a lateral head film, which is available as a pre-treatment record. However, in several instances, optimal treatment timing is to be delayed until after the diagnosis, making necessary a later re-evaluation of the growth phase. Moreover, the cervical vertebrae might be partially covered by the protection collar, which would be necessary to reduce radiation exposure (28). Even though the radiographical recording of the hand and wrist has been shown to be safer in terms of radiation exposure (28), this method requires anyway additional X-ray exposure of a hand and wrist as a whole, other than a dedicated X-ray machine. Besides, re-execution of either a lateral head cephalogram or a handand-wrist film for a re-evaluation of growth phases is not indicated according to the most recent guidelines (29).

In the present study, the mean chronological ages at which both females and males reached the pubertal growth spurt, as recorded by the MPS3 or CS3 (Fig. 2), are comparable with previously reported data $(3,5,30)$.

The correlation coefficient seen in the present study between the MPM and CVM methods is very similar to that above 0.94 obtained in a previous investigation (16) that was based on the maturation staging of the middle phalanx of the third finger proposed by Hägg and Taranger (5).
Moreover, the weighted $\kappa$ coefficients retrieved herein were very high up to 0.88 denoting a very good overall agreement between the two maturational methods. Again, this analysis was missed in the previous studies (14-16).

In the present study, about $78 \%$ of agreement was found, and disagreements showed mostly a single stage apart. The disagreement seen herein may be explained by the fine transitional morphological changes in either third middle phalanx or cervical vertebrae. However, good agreements between the maturational methods were those for stages 3 , in which all the diagnostic parameters showed a high performance of the MPM method in the identification of the corresponding CVM staging (Table 2). Therefore, events responsible for the onset of the pubertal growth spurt, that is, hormonal changes (25), would induce concomitant morphological changes in both the third middle phalanx and cervical vertebrae.

Satisfactory diagnostic agreement with accuracy and positive LHRs values above $90 \%$ and $10 \%$, respectively, for each MPM stage in the identification of the corresponding CVM stage (Table 2). However, the calculation of the accuracy and the positive LHRs takes into account both the identification of true positive and true negative cases. Therefore, when dealing with several possible clustering, an important diagnostic parameter is the positive predictive value that gives an indication of the capability of a given MPM stage in the identification of the corresponding CVM stage, irrespective of the number of true negative cases belonging to the other stages. By analyzing the positive predictive values, in combination with the frequency distributions of the maturational staging, a general tendency for the MPM to reach the stage 6 slightly earlier than the CVM is evident (Table 2). The concept that small morphological changes at the third middle phalanx may be better detected than those at the cervical vertebrae may also be responsible for this evidence. Therefore, from a clinical perspective, a safe diagnosis of the attainment of the post-pubertal growth phase especially in males should rely on the attainment of MPS6, rather than MPS5. 
Further studies on the correlation/diagnostic performance of the present MPM method with statural or mandibular growth, or even to other hand-wrist maturation methods, are warranted.

\section{Clinical implications}

The availability of a radiographical method based on a very minimal radiation exposure appears to be a valuable tool in clinical practice. The MPM method appears to be a valid indicator of the onset of the pubertal growth spurt in individual subjects and may therefore find wide applications for planning treatment timing for functional treatments for skeletal class II or III (1) and constricted maxilla (31). Finally, the MPM method is of easy execution and interpretation and may be performed in any clinical setting with minimal instrumentation. This method may also be complementary when the CVM staging would be uncertain or not derivable form a lateral cephalogram.

\section{Conclusions}

1) The MPM and CVM methods show an overall satisfactorily diagnostic agreement; 2) good agreement for stages 3 that corresponds to the onset of the pubertal growth spurt; and 3) a slight disagreement at stage 5 , in which the third middle phalanx appears to mature earlier than the cervical vertebrae.

\section{Clinical relevance}

Individual monitoring of the growth phase, with particular regard to the onset of the pubertal growth spurt, has been advocated to obtain predictable treatment effects when dealing with skeletal malocclusions. Although slight differences exist, when compared with the cervical vertebral method, the maturational staging of the middle phalanx of the third finger appears to be a valid indicator of the onset and of the end of the pubertal growth spurt in individual subjects. When a lateral head film is not available or not clear in the cervical area, the middle phalanx maturation method may be used as a valid alternative method.

\section{Competing interest}

The authors declare that they have no competing financial interests.

Acknowledgements: The authors are deeply grateful to Dr. Nicolò Favaretto (University of Trieste) and Dr. Serena Cavuoti (Second University of Naples) for their support in screening the radiographical records.

\section{References}

1. Baccetti T, Franchi L, McNamara JAJ. The cervical vertebral maturation (CVM) method for the assessment of optimal treatment timing in dentofacial orthopedics. Semin Orthod 2005;11:119-29.

2. Petrovic A, Stutzmann J, Lavergne J. Mechanism of craniofacial growth and modus operandi of functional appliances: a cell-level and cybernetic approach to orthodontic decision making. In: Carlson DS, editor. Craniofacial Growth Theory and Orthodontic Treatment Monograph 23 Craniofacial Growth Series. Ann Arbor, MI: Center for Human Growth and Development, University of Michigan; 1990. pp. 13-74.
3. Fishman LS. Radiographic evaluation of skeletal maturation. A clinically oriented method based on handwrist films. Angle Orthod 1982;52:88112.

4. Greulich WW, Pyle SI. Radiographic Atlas of Skeletal Development of the Hand and Wrist, 2nd edn. Stanford, CA: Stanford University Press; 1959.

5. Hägg U, Taranger J. Maturation indicators and the pubertal growth spurt. Am J Orthod 1982;82: 299-309.

6. Perinetti G, Baccetti T, Contardo L, Di Lenarda R. Gingival crevicular fluid alkaline phosphatase activity as a non-invasive biomarker of skeletal maturation. Orthod Craniofac Res 2011;14:44-50.
7. Flores-Mir C, Nebbe B, Major PW. Use of skeletal maturation based on hand-wrist radiographic analysis as a predictor of facial growth: a systematic review. Angle Orthod 2004;74:118-24.

8. Santiago RC, de Miranda Costa LF, Vitral RW, Fraga MR, Bolognese AM, Maia LC. Cervical vertebral maturation as a biologic indicator of skeletal maturity. Angle Orthod 2012;82:1123-31.

9. Beit P, Peltomaki T, Schatzle M, Signorelli L, Patcas R. Evaluating the agreement of skeletal age assessment based on hand-wrist and cervical vertebrae radiography. Am J Orthod Dentofacial Orthop 2013;144:838-47. 
10. Hassel B, Farman AG. Skeletal maturation evaluation using cervical vertebrae. Am J Orthod Dentofacial Orthop 1995;107:58-66.

11. Martina R, Cioffi I, Galeotti A, Tagliaferri R, Cimino R, Michelotti A et al. Efficacy of the Sander bitejumping appliance in growing patients with mandibular retrusion: a randomized controlled trial. Orthod Craniofac Res 2013;16:116-26.

12. Uysal T, Ramoglu SI, Basciftci FA, Sari Z. Chronologic age and skeletal maturation of the cervical vertebrae and hand-wrist: is there a relationship? Am J Orthod Dentofacial Orthop 2006;130:622-8.

13. Perinetti G, Contardo L, Gabrieli P, Baccetti T, Di Lenarda R. Diagnostic performance of dental maturity for identification of skeletal maturation phase. Eur J Orthod 2012;34:487-92.

14. Rajagopal R, Kansal S. A comparison of modified MP3 stages and the cervical vertebrae as growth indicators. J Clin Orthod 2002;36:398-406.

15. Ozer T, Kama JD, Ozer SY. A practical method for determining pubertal growth spurt. Am J Orthod Dentofacial Orthop 2006;130:131 e131-136.

16. Wong RW, Alkhal HA, Rabie AB. Use of cervical vertebral maturation to determine skeletal age. Am J Orthod Dentofacial Orthop 2009;136:484 e481-486; discussion 484-485.

17. Gwet KL. Inter-Rater Reliability Discussion Corner. http://agreestat. com/blog_irr/sample_size_determination.html. Last accessed August 9, 2012; 2010.
18. Abdel-Kader HM. The reliability of dental x-ray film in assessment of MP3 stages of the pubertal growth spurt. Am J Orthod Dentofacial Orthop 1998;114:427-9.

19. Brenner H, Kliebsch U. Dependence of weighted kappa coefficients on the number of categories. Epidemiology 1996;7:199-202.

20. Greenhalgh T. Advertisements for donepezil. More convincing evidence of efficacy needs to be cited. $B M J$ 1997;315:1623; author reply 1624.

21. Deeks JJ, Altman DG. Diagnostic tests 4: likelihood ratios. $B M J$ 2004;329:168-9.

22. Franchi L, Baccetti T, McNamara JA Jr. Mandibular growth as related to cervical vertebral maturation and body height. Am J Orthod Dentofacial Orthop 2000;118:335-40.

23. Soegiharto BM, Moles DR, Cunningham SJ. Discriminatory ability of the skeletal maturation index and the cervical vertebrae maturation index in detecting peak pubertal growth in Indonesian and white subjects with receiver operating characteristics analysis. Am J Orthod Dentofacial Orthop 2008;134:227-37.

24. Perinetti G, Franchi L, Castaldo A, Contardo L. Gingival crevicular fluid protein content and alkaline phosphatase activity in relation to pubertal growth phase. Angle Orthod 2012;82:1047-52.

25. Masoud M, Masoud I, Kent RL Jr, Gowharji N, Cohen LE. Assessing skeletal maturity by using blood spot insulin-like growth factor I
(IGF-I) testing. Am J Orthod Dentofacial Orthop 2008;134:209-16.

26. Gabriel DB, Southard KA, Qian F, Marshall SD, Franciscus RG, Southard TE. Cervical vertebrae maturation method: poor reproducibility. Am J Orthod Dentofacial Orthop 2009;136:478 e471-477; discussion 478-480.

27. Nestman TS, Marshall SD, Qian F, Holton N, Franciscus RG, Southard TE. Cervical vertebrae maturation method morphologic criteria: poor reproducibility. Am J Orthod Dentofacial Orthop 2011;140:182-8.

28. Patcas R, Signorelli L, Peltomaki T, Schatzle M. Is the use of the cervical vertebrae maturation method justified to determine skeletal age? A comparison of radiation dose of two strategies for skeletal age estimation. Eur J Orthod 2012;35:604-9.

29. Isaacson KG, Thom AR, Horner K, Whaites E. Orthodontic Radiographs - Guidelines for the Use of Radiographs in Clinical Orthodontics, 3rd edn. London: British Orthodontic Society; 2008.

30. Soegiharto BM, Cunningham SJ, Moles DR. Skeletal maturation in Indonesian and white children assessed with hand-wrist and cervical vertebrae methods. Am J Orthod Dentofacial Orthop 2008;134:217-26.

31. Franchi L, Baccetti T, McNamara JA. Postpubertal assessment of treatment timing for maxillary expansion and protraction therapy followed by fixed appliances. Am J Orthod Dentofacial Orthop 2004;126:555-68. 